\title{
Construir una voz propia: la escritura de la sección Introducción en tesinas de licenciaturas
}

\author{
Acquiring a Voice of Their Own: Writing Features of Introduction Sections in Degree \\ Thesis
}

\author{
Yamila Daniela Saldivia Levicoy, Mónica Musci \\ daniela.sdvia@hotmail.com,mmusci@uarg.unpa.edu.ar \\ UNPA-ICIC-UARG
}

Recibido: 01/03/2019. Aceptado: 04/07/2019

\begin{abstract}
RESUMEN
Hacia el interior de las comunidades discursivas disciplinares los escritores hablan a sus colegas de modos reconocibles y aceptables, modelando sus acciones en función de la comprensión de sus lectores (Hyland, 2004). Para ser considerados miembros de esas comunidades, un primer requisito es 'escribir al modo de la academia', respetando ciertos modos de decir que los destinatarios reconocerán como aceptables. En este proceso, la escritura de la tesina de grado suele adquirir la cualidad de un verdadero 'rito de pasaje' en el cual los enunciadores logran emitir su propia voz, diferenciarla de otras con las que dialogan y discuten, para emitir comentarios, juicios, razonamientos y conclusiones.

Guiadas por el objetivo general de describir los procedimientos a través de los cuales los estudiantes de grado construyen en su escritura una identidad académica, el presente trabajo muestra algunos resultados de la exploración de la sección Introducción de tesinas de licenciatura de cuatro carreras de la UNPA, focalizando la atención en los movimientos retóricos con que las organizan, en las marcas de personalización y despersonalización y en el manejo de la intertextualidad en sus escritos. Los resultados muestran por una parte, que los alumnos escritores manejan algunas de estas herramientas con cierta destreza (como la citación bibliográfica) y son cautelosos en la exhibición de la subjetividad. Por otra parte, la indagación pone de manifiesto las dificultades de estos escritores noveles para relacionar su contribución específica con el campo disciplinar al que pertenecen.
\end{abstract}

Palabras clave: Ethos académico; movimientos retóricos; personalización; citas bibliográficas; tesinas.

\begin{abstract}
Within disciplinary discursive communities writers talk to their colleagues in recognizable and acceptable ways, designing their actions based on the understanding of their readers (Hyland, 2004). In order to be considered a member of these communities, the first requirement is to "write in an academic manner", respecting certain ways of saying that the public will recognize as acceptable. During this process, the task of writing a degree thesis usually becomes a real "ritual passage" in which enunciators acquire a voice of their own, distinguishing it from other voices with which they dialogue and discuss, to express remarks, opinions, reasons and conclusions. With the aim of describing the procedures by which degree students build an academic identity in their writing, this paper shows some results of the
\end{abstract}


exploration of Introduction sections in degree thesis of four different careers in UNPA, focusing on the rhetoric moves that organize them, the marks of personalization and depersonalization and the managing of individuality in their writings. The results show that student-writers have considerable skills in handling some of these tools (like bibliographic citation) and that they are cautious in the exhibition of subjectivity. On the other hand, the inquiry brings to light the difficulties these novel writers experience when relating their specific contribution to the disciplinary field to which they belong.

Key words: Academic ethos; rethoric moves; personalization; bibliographic citation; thesis.

\section{INTRODUCCIÓN}

Dentro de los estudios actuales de Lingüística aplicada, la reflexión sobre los textos académicos, las prácticas de lectura y escritura en la educación superior y los modos de decir y argumentar propios de cada disciplina, constituyen un campo en expansión. Esta reflexión está alimentada, urgida y exigida por la demanda concreta de intervenciones que ayuden a los estudiantes de grado y posgrado a adquirir la experticia necesaria para incorporarse a las comunidades discursivas de sus respectivas disciplinas a través de la producción escrita. Leer y escribir son prácticas lingüísticas que no se aprenden de una vez y para siempre sino a lo largo de toda la vida académica y profesional porque cada campo del saber se rige por normas discursivas propias, codificadas por convenciones de géneros, tradiciones y modos de decir diferenciados, a las que se accede a través de aprendizajes específicos. Las investigaciones de las últimas décadas demuestran que la adquisición de las competencias necesarias para dominar los modos de leer y escribir propios de los estudios superiores "no se adquieren de manera espontánea sino que requieren de un proceso instruccional intencionalmente dirigido a promoverlas" (Castelló, 2009).

Gran parte de los estudios que toman como objeto los textos escritos por los alumnos señalan sus carencias y problemas para participar de la vida universitaria y avanzar en sus trayectos académicos. Otra línea de investigación actual (Riera, 2015) pone el foco en la descripción de la escritura académica de los alumnos como un objeto particular de estudio que merece la pena describir y analizar. La perspectiva elegida en este trabajo no es la que señala la carencia, sino aquella que se interesa por los escritos 'exitosos' de los alumnos universitarios, los que son evaluados por los miembros más antiguos de la comunidad académica como buenas producciones y pueden considerarse un aceptable escalón intermedio en el camino de aprendices a expertos. Entre estos textos se encuentran, evidentemente, las tesinas de licenciatura ${ }^{1}$ que, resultado de arduos esfuerzos individuales, representan un modo efectivo en que los alumnos, en el umbral de los estudios de grado, se apropian eficazmente de la escritura académica como instrumento para convertirse en miembros de las comunidades disciplinares con una voz propia.

Hacia el interior de las comunidades discursivas disciplinares los escritores hablan a sus colegas de modos reconocibles y aceptables, modelando sus acciones en función de la comprensión de sus lectores (Hyland, 2004). Para ser considerados miembros de esas comunidades, un primer requisito es 'escribir al modo de la academia', respetando ciertas formas de decir que los destinatarios reconocerán como admisibles. Como otras prácticas sociales, la escritura académica es una actividad históricamente situada anclada en

\footnotetext{
${ }^{1}$ Usamos la denominación 'tesina' para el trabajo final de carreras de grado, aunque en los documentos oficiales suele aparecer la etiqueta 'tesis', que reservamos para la escritura de posgrado
} 
representaciones acerca del lenguaje, los roles discursivos y los textos (Di Stéfano, 2017). Esta perspectiva revela el carácter convencional de las reglas y exigencias de las "tribus" académicas (Hyland, 2004) de las cuales los escritores inexpertos deben apropiarse para poder construir una voz propia y entrar en diálogo con las demás voces de su campo científico. En este proceso, la escritura de la tesina de grado suele adquirir la cualidad de un verdadero 'rito de pasaje' en el que los enunciadores intentan dar forma a su propia voz, diferenciarla de otras con las que dialogan y discuten, para emitir comentarios, juicios, razonamientos y conclusiones.

Dentro de los géneros académicos, las tesinas de licenciatura han sido descriptas en sus aspectos estructurales y enunciativos en el ámbito de la lengua española por Tamola de Spiegel (2005). Existen también varios estudios acerca de un género académico similar, las monografías escritas por los estudiantes universitarios (Navarro y Moris, 2012; Moris y Pérez, 2014; Gabbiani y Orlando, 2016), en los que se han señalado rasgos estructurales y estilísticos específicos que obedecen a convenciones disciplinares, no siempre explícitas. Dentro de un proyecto de investigación más general dedicado a la descripción de las características particulares de la escritura académica de los estudiantes universitarios ${ }^{2}$, el presente trabajo pone el foco en las estrategias a través de las cuales los alumnos 'toman' la palabra para presentar sus propios puntos de vista ante la comunidad disciplinar en la que aspiran a insertarse, es decir, construyen una voz de autor, aspecto central en los escritos académicos. Esta voz es producto de estrategias de textualización, las más relevantes de las cuales son la organización de la información, la personalización, la despersonalización y el aparato de referencias bibliográficas, herramientas que presentan al autor "discursivamente como un locutor que investiga, selecciona y ordena" datos, material bibliográfico, y al mismo tiempo, propone una interpretación (Moris y Pérez, 2014: 224).

Creemos que la indagación en los modos en que los escritores noveles despliegan estas estrategias en pos del objetivo de asumir una voz propia e intentan conformar un ethos académico-disciplinar ${ }^{3}$, es un aspecto central que puede iluminar ciertos procesos de pensamiento y de escritura que suelen ser poco visibles para los estudiantes.

Realizamos nuestra indagación a partir de tres ejes: la organización retórica de las introducciones, la tensión objetividad-subjetividad y la gestión del aparato de citación bibliográfica. Los resultados muestran algunas diferencias entre las disciplinas, lo que sugiere que los alumnos escritores son sensibles a las convenciones que sus ámbitos de conocimiento les imponen.

Los resultados de este proyecto pueden constituir un insumo importante para el diseño de dispositivos didácticos que favorezcan la escritura en la universidad. En primer lugar, la reflexión sobre estos procesos será fundamental para la caracterización del género 'tesina' de

\footnotetext{
${ }^{2}$ PI 29/A370. La escritura académica de los estudiantes universitarios. Un proceso de apropiación discursiva. Dir. Mónica Musci. UNPA. 2016-2018.

${ }^{3}$ La noción de ethos proviene de la Retórica Clásica y ha sido retomada por el Análisis del Discurso. Señala Amossy (1999) que "toda toma de palabra implica la construcción de una imagen de sí mismo. A tal efecto, no es necesario que el locutor trace su retrato, detalle sus cualidades ni aún que hable explícitamente de sí mismo. $\mathrm{Su}$ estilo, sus competencias lingüísticas y enciclopédicas, sus creencias implícitas alcanzan para dar una representación de su persona. (...) Deliberadamente o no, el locutor efectúa de este modo en su discurso, una presentación de sí mismo." Según Maingueneau (2002) "No se trata de una representación estática o bien delimitada, sino sobre todo de una forma dinámica, construida por el destinatario a través del movimiento mismo de la palabra del locutor. (...) la cuestión del ethos está ligada a la construcción de la identidad" (Maingueneau, 2002).
} 
las carreras cuyos trabajos constituyen nuestro corpus, ya que a partir del conocimiento producido, podrán realizar un mejor acompañamiento del proceso de escritura que llevan a cabo los alumnos (adecuación de consignas, anticipación de problemas recurrentes, ajuste de etapas y tiempos asignados a cada una) y a la vez se contribuirá a la generación de una mayor conciencia metadiscursiva en los docentes.

El trabajo se estructura de la siguiente manera: en primer lugar, presentaremos el corpus y describiremos la metodología de investigación para cada uno de los ejes en los que indagamos. A continuación abordaremos cada uno de ellos: en primer lugar los movimientos retóricos en las introducciones, en segundo lugar las estrategias de personalización y despersonalización, en tercer lugar los modos de gestión del discurso ajeno. En cada apartado presentaremos un breve marco teórico, los resultados y el análisis. Finalmente expondremos las principales conclusiones de este trabajo y trazaremos algunas líneas futuras de investigación.

\section{DESCRIPCIÓN DEL CORPUS Y METODOLOGÍA}

El corpus de análisis de este trabajo está compuesto por las introducciones de seis tesinas de licenciatura aprobadas en el período 2013-2015, que pertenecen a las siguientes carreras de grado: dos de la licenciatura en Comunicación Social, dos de la licenciatura en Trabajo Social, una de la licenciatura en Letras y una de la licenciatura en Geografía. Todas ellas, pertenecientes a la Universidad Nacional de la Patagonia Austral, Unidad Académica Río Gallegos (Tabla 1).

\section{Tabla 1. Corpus de análisis}

\begin{tabular}{|c|c|c|}
\hline Carreras $^{4}$ & Número de introducciones & Año de la tesina \\
\hline Licenciatura en & 2 & 2015 \\
Comunicación Social & & 2014 \\
\hline Licenciatura en Trabajo & 2 & 2014 \\
Social & 1 & 2014 \\
\hline Licenciatura en Letras & 1 & 2014 \\
\hline Licenciatura en Geografía & & 2013 \\
\hline
\end{tabular}

Nuestra atención está centrada en la sección Introducción, ya que es el fragmento donde los alumnos tesistas despliegan mayor cantidad de estrategias que sostienen la construcción de una voz propia. Nos detenemos a identificar y describir tres de ellas: los movimientos retóricos a través de los cuales los escritores organizan y hacen progresar la información; los modos gramaticales de personalización y despersonalización, a través de los cuales el autor maneja la gestión de la subjetividad en el texto y por último, los mecanismos de citación, por medio de los cuales el enunciador entabla el diálogo con otras voces dentro del campo disciplinar. Estas son dimensiones que aparecen casi obligatoriamente en la parte introductoria del trabajo final, cuyo carácter informativo, retórico y referencial puede registrarse en nuestro conjunto de tesinas. Las Introducciones tienen una extensión promedio de 3 páginas.

\footnotetext{
${ }^{4}$ En adelante, CS (Comunicación Social), TS (Trabajo Social), L (Letras), G (Geografía).
} 
Esta investigación es descriptiva, con intenciones, además, de llegar a establecer algún tipo de correlación entre variables, en forma provisoria debido al tamaño de la muestra. Se trata de una investigación aplicada, que trabaja preponderantemente con información de naturaleza cualitativa, aun cuando se utilizarán algunas cuantificaciones para el rastreo de tendencias generales. Analizaremos textos escritos por los alumnos tal cual ocurren, sin manipulación deliberada de variables o intervenciones de nuestra parte. En tal sentido se trata de una investigación no experimental.

Abordaremos el análisis de los tres ejes mencionados con diferentes aproximaciones:

- Para el análisis de los movimientos retóricos tomaremos como base el modelo CARS (Swales, 1990) y la reformulación para artículos de investigación y tesis en español que realizan Sabaj et al. (2011) y Difabio de Anglat y Álvarez, (2015). En contraste con estas esquematizaciones describiremos los movimientos que aparecen efectivamente en nuestro corpus. Si bien el modelo original estaba orientado a explicar la estructura de las introducciones de artículos de investigación (AI), la noción de movimiento retórico ha resultado sumamente productiva en el campo de la enseñanza de la escritura científica y ha sido una herramienta útil para de escribir otras secciones del mismo género e incluso otros géneros científicos y académicos. (Cubo de Severino, 2005).

- Para el análisis de cómo los escritores noveles gestionan la subjetividad en sus textos recurrimos a un abordaje cuantitativo que considera el peso relativo de dos grandes variables a través de la expresión de los porcentajes de ocurrencia: estrategias de despersonalización (con sus diferentes realizaciones: pasivas e impersonales con se, uso del infinitivo, metonimia, uso de la $3^{\circ}$ persona) y estrategias de personalización (usos de la primera persona coincidente o primera persona plural).

- Para el análisis de la gestión bibliográfica, es decir, el análisis de los tipos de citas, tenemos en cuenta la clasificación tradicional de citas directas e indirectas (Reyes, 1995) y la distinción entre citas integradas y no integradas (Swales, 1990). A partir del cruce de ambas taxonomías obtenemos cuatro modos de incluir las voces ajenas en el propio discurso y a través de una cuantificación del peso porcentual de cada estrategia en el total de ocurrencias, intentamos establecer algunas correlaciones entre tipos de citas y campos de conocimiento.

\section{RESULTADOS, ANÁLISIS Y DISCUSIÓN}

\section{A. MOVIMIENTOS RETÓRICOS: LA ORGANIZACIÓN Y PROGRESIÓN DE LA INFORMACIÓN}

\section{A.1. MARCO TEÓRICO}

Los estudiantes de carreras de grado deben llevar a cabo la tarea de escribir una tesina para obtener el título de licenciado/a. Como todos los textos en interacción comunicativa, las tesinas de licenciatura contribuyen a la realización de metas comunicativas sociales y objetivos individuales, así como a la constitución de relaciones sociales (Tamola de Spiegel, 2005). Entre las habilidades que los alumnos deben desplegar para desarrollar esta tarea se encuentra la competencia para elaborar discursivamente una presentación sucinta pero completa de un proceso de investigación, propósito comunicativo que sostiene la introducción de un trabajo académico (Swales, 1990:57; Puiatti de Gómez y Castro de Castillo, 2011:54). 
En la Introducción de estas producciones, los escritores deben moverse entre dos polos: desde los propósitos generales del campo científico hasta los detalles de su investigación particular. En este proceso recurren a los movimientos retóricos a través de los cuales organizan el texto de modo coherente y cohesivo. El trabajo de escribir siguiendo pasos con fines comunicativos, representa una demanda dual en tanto el texto debe mostrar una jerarquización temática, pero al mismo tiempo, debe "lograr la progresión lineal, oración por oración" (Difabio de Anglat y Álvarez, 2015:1).

La noción central que tomamos para este análisis: Movimiento Retórico (en adelante, MR) fue propuesta por el lingüista inglés John Swales (1990) en su artículo Research articles in English, que la utiliza para dar cuenta de la estructura de las Introducciones de Artículos Científicos en inglés. Swales considera que el propósito de la Introducción es crear y ocupar un espacio de investigación: Create A Research Space (CARS, para su sigla en inglés). Un $\mathrm{MR}$ es "la expresión de un propósito comunicativo que se asocia a un fragmento textual, y que contribuye al logro del propósito global de un género" (Sabaj et al. 2011:247). Este movimiento (move) está constituido por pasos (steps) que concretan el medio retórico de la realización de la movida (Difabio de Angat y Álvarez, 2015:1). Sin embargo, según la opinión de Sabaj y otros (2011) la forma en que los pasos constituyen un MR es indefinida, para ellos tienen ambas nociones la misma naturaleza, en tanto que por medio de estos se realizan propósitos comunicativos ${ }^{5}$. Nosotros tomaremos la postura que señala que los pasos son las diferentes realizaciones de cada movimiento (Difabio de Anglat y Álvarez, 2015).

El modelo ha resultado sumamente productivo en el campo de la enseñanza de la escritura científica. Sabaj Meruane et al (2011) han señalado que aún cuando fue elaborado en base a la producción científica en inglés, es una herramienta que se adecúa perfectamente para ser aplicada en el ámbito de la escritura científica en español. Por otro lado, si bien el modelo original intentaba explicar la estructura de las introducciones, este marco teóricometodológico ha sido extendido para describir otras secciones de los artículos científicos y la noción de MR ha sido una herramienta útil para estudiar también otros géneros científicos y académicos. Es importante destacar que según Swales, el modelo es flexible en relación con los modos de realización lingüística de los pasos y movimientos, ya que se trata de unidades funcionales y no formales (un paso puede realizarse a través de una cláusula o de varias). Además, un mismo paso puede aparecer en diferentes secciones del artículo ya que a lo largo del texto se expresan cíclicamente ciertos propósitos comunicativos. El modelo de Swales (1990) propone que las introducciones de los AI se componen de tres movimientos (Tabla 2).

Tabla 2. Los movimientos retóricos del Modelo CARS, Swales $(1990,2004)$

1) Establecer el campo

2) Establecer el espacio vacante o también llamado 'nicho'

3) Ocupar el 'nicho' vacío o presentar el trabajo.

Cada uno de estos movimientos está compuesto por pasos (Tabla 3). Tomamos el modelo original con algunas modificaciones propuestas para el género tesis por Di Fabio de Anglat y Álvarez (2015) que a su vez las toman de las propuestas de Jara Solar (2013) y Bunton (2002).

\footnotetext{
${ }^{5}$ Los autores optan por la etiqueta 'movida retórica', utilizada por la Escuela de Valparaíso, que neutraliza la diferencia de escala y se aplica tanto a Movimientos como a Pasos.
} 
Tabla 3. Pasos del Modelo CARS, Swales (1990) reformulado por Difabio de Anglat y Álvarez (2015)

\begin{tabular}{|l|}
\hline Movimiento 1. Establecer el campo \\
1.a Justificar la importancia del tema \\
1.b Presentar generalizaciones sobre el tema \\
1.c Revisar investigación previas \\
1.d Definir conceptos importantes \\
Movimiento 2. Establecer el espacio vacante o también llamado 'nicho' ${ }^{6}$ \\
2.a Indicar lagunas en la investigación \\
2.b Indicar problemas o necesidades \\
2.c Formular preguntas \\
2.d Continuar la tradición \\
Movimiento 3. Ocupar el 'nicho' vacío o presentar el trabajo. \\
3.a Formular propósitos u objetivos, generales y específicos \\
3.b Justificar la investigación \\
3.c Describir características importantes de la investigación \\
3.d Anunciar resultados y hallazgos \\
3.e Anunciar aportes y proyecciones de la investigación \\
3.f Explicar la estructura textual adoptada en la tesis \\
\hline
\end{tabular}

\section{A.2. MOVIMIENTOS Y PASOS EN LAS INTRODUCCIONES DEL CORPUS}

Como es sabido, las tesinas de licenciatura transmiten la información a partir de un tema de interés en el que se observa una laguna o problema que debe resolverse. Al analizar nuestro corpus nos preguntamos qué movimientos y pasos pueden homologarse a los descriptos por el modelo. En la introducción de estos trabajos veremos que los alumnos utilizan estrategias para mostrar, por ejemplo, la relevancia del tema abordado, los propósitos, las características más importantes de la investigación, entre otros pasos. Guiándonos por las tablas 2 y 3, podemos observar que el primer movimiento, Establecer el campo, aparece en las Introducciones de CS, G y L a través de los pasos "presentar generalizaciones sobre el tema", "justificar la importancia del tema", "definir conceptos importantes". En contraste, observamos que no hay pasos que realicen este primer movimiento en las introducciones de la carrera de TS. En ninguna de nuestras introducciones aparece el Paso 3: "Revisa investigaciones previas". En la Tabla 4 observamos ejemplos de las realizaciones de estos pasos en las introducciones que analizamos:

Tabla 4. Movimiento 1 en las Introducciones de Tesinas: Establecer el campo

\begin{tabular}{|c|c|}
\hline $\begin{array}{l}\text { Paso 1. Justificar la importancia del } \\
\text { tema }\end{array}$ & $\begin{array}{l}\text { (1)La temática abordada se inserta en uno de los } \\
\text { aspectos ambientales más importantes a nivel } \\
\text { mundial: la disponibilidad del recuerdo hídrico... } \\
(\boldsymbol{G})\end{array}$ \\
\hline $\begin{array}{l}\text { Paso 2. Presentar generalizaciones sobre } \\
\text { el tema }\end{array}$ & $\begin{array}{l}\text { (2) En este apartado se hizo necesario aclarar que el } \\
\text { Programa CI fue creador por decreto n } 459 / 10 \text { bajo } \\
\text { el nombre "Programa Conectar Igualdad.Com.Ar", } \\
\text { según los términos de este decreto se planteó el } \\
\text { modo de implementación y sus fundamentos" (CS2) }\end{array}$ \\
\hline $\begin{array}{l}\text { Paso 4. Definir conceptos importantes a } \\
\text { través de las citas de autoridad. }\end{array}$ & $\begin{array}{l}\text { (3) ... el erotismo se define por "la independencia } \\
\text { del goce erótico respecto de la reproducción } \\
\text { considerada como fin, no por ello es menos cierto } \\
\text { que el sentido fundamental de la reproducción es la } \\
\text { clave del erotismo" (Bataille, 2009:15) (L) }\end{array}$ \\
\hline
\end{tabular}

\footnotetext{
${ }^{6}$ Swales (1990) considera que estos pasos son opcionales.
} 
En relación con el segundo movimiento, Establecer el nicho, encontramos que se realiza en algunos casos a través de la opción tres, Formular preguntas, es decir, el tesista formula preguntas de investigación abiertas o cerradas como punto de partida de sus planteos (Difabio de Anglat y Álvarez, 2015).

(4) ¿Por qué la poesía erótica atraviesa y sostiene la poética dariana manifestándose allí un campo de batalla signado por tensiones, contradicciones, antítesis, pero también ambigüedades $(\boldsymbol{L})$.

(5) ¿Cuáles fueron los principales aspectos y cambios en la publicidad gráfica de las campañas electorales en Santa Cruz? (CS1)

Este paso solo puede observarse en las tesinas de L y CS. No aparecen los otros pasos del movimiento en nuestro corpus, es decir, los escritores prestan poca atención a señalar un vacío de investigación en el campo, un aspecto no trabajado que podría mostrar la necesidad de su propia indagación.

Del movimiento tres, Ocupar el nicho o Presentar el trabajo, podemos señalar que se realiza a través de algunos de los cuatro pasos, es decir, "Formular propósitos u objetivos generales y específicos"; "Justificar la investigación"; "Describir características importantes de la investigación" y "Anunciar resultados y hallazgos". Debemos señalar que es el movimiento más utilizado por los autores de nuestro corpus, no falta en ninguna de las tesinas (Tabla 5).

Tabla 5. Movimiento 3 en las Introducciones de Tesinas: Ocupar el nicho

\begin{tabular}{|l|l|}
\hline $\begin{array}{l}3.1 \text { Formular propósitos u objetivos, } \\
\text { generales y específicos }\end{array}$ & $\begin{array}{l}\text { (6) En el presente trabajo, postulamos que la poesía } \\
\text { erótica atraviesa la obra de Rubén Dario, } \\
\text { destacando la desmitificación de la mujer como } \\
\text { agente pasivo en el acto amoroso... (L) }\end{array}$ \\
\hline 3.2 Justificar la investigación & $\begin{array}{l}\text { (7) ...la presente tesis aporta información de utilidad } \\
\text { para la gestión de los recursos hídricos superficiales } \\
\text { en la ciudad de Ushuaia en relación al crecimiento } \\
\text { poblacional estimado para las próximas décadas. } \\
\text { (G) }\end{array}$ \\
\hline $\begin{array}{l}\text { 3.3 Describir características importantes } \\
\text { de la investigación }\end{array}$ & $\begin{array}{l}\text { (8) El trabajo a desarrollar se encuentra } \\
\text { conformado por cinco grandes momentos. En un } \\
\text { primer lugar se enfocará sobre la experiencia, } \\
\text { delimitando adecuadamente el objeto, objetivos y } \\
\text { ejes de la sistematización, así como la metodología } \\
\text { acorde a la complejidad del proceso de desarrollo, y } \\
\text { las fuentes de recuperación de la experiencia. (TS1) }\end{array}$ \\
\hline 3.4 Anunciar resultados y hallazgos & $\begin{array}{l}\text { (9) Entonces, sobre ese sentido observado e distintas } \\
\text { categorías, se señaló que cuanto mayor sean los } \\
\text { sentidos expresados - en las distintas categorías de } \\
\text { este estudio- mayor serála apropiación. (CS2) }\end{array}$ \\
\hline
\end{tabular}

\section{A.3. ANÁLISIS DE LOS RESULTADOS: INCORPORARSE (O NO) AL CAMPO ACADÉMICO-DISCIPLINAR}

La indagación nos permite señalar dos cuestiones: la primera, que los autores efectivamente utilizan algunos movimientos retóricos típicos, en especial, el tercero, que atañe directamente al propio trabajo: Ocupar el nicho de investigación; la segunda cuestión, que se verifica un escaso empleo (que llega a ser nulo en TS) de los movimientos uno y dos. Prevalece en el 
corpus el tercer movimiento, sobre todo los pasos de formulación de objetivos y descripción de las características más importantes de la investigación.

Esta estructura retórica 'incompleta' otorga a las tesinas del corpus un carácter de investigación particular que no muestra con claridad la ligazón que tienen con el saber general del campo de conocimiento al que pertenecen. La mayoría de estas introducciones no tienen la forma de 'embudo' característica que delinea el camino de lo general a lo específico sino que introducen rápidamente el asunto que se investiga. En otras palabras, el trabajo que se presenta no aparece inserto en la corriente general de la empresa científica, sino que se plantea como una indagación que responde a demandas más cercanas (la más inmediata de las cuales, conjeturamos, es la aprobación de un espacio curricular concreto).

Aquí se evidencia una debilidad en la construcción retórica que hace que un escritor piense en el lector como alguien al que tiene que conducir de un tema general, bien conocido, al tema en particular. Por otra parte, el movimiento de señalar el vacío de investigación, encontrar el nicho, es un movimiento altamente persuasivo en el que el escritor busca convencer al lector de que su propósito de investigación y escritura es necesario para llenar un vacío de conocimiento en el campo, para justificar el valor de que alguien dedique tiempo y esfuerzo a realizar esa investigación. En cambio, la mayoría de estos alumnos escritores van 'derecho al grano' en general, es decir, no dedican un esfuerzo a convencer al lector de que la tarea que proponen contribuye al propósito general de la ciencia y es necesaria para el campo de conocimiento. Es posible que no visualicen su propia contribución de esta manera, sino como la respuesta a una tarea de escritura asignada por la cátedra. Este es un rasgo que puede construirse estimulando a los escritores novatos a referenciar su tarea de investigación en las líneas generales de un campo de conocimiento.

\section{B. MOSTRARSE U OCULTARSE: MARCAS DE SUBJETIVIDAD EN LAS TESINAS}

\section{B.1. MARCO TEÓRICO}

En la escritura de una tesina de licenciatura, los alumnos escritores deben necesariamente tomar posición respecto a aquello analizado u observado y es allí donde se va consolidando la voz de autor. En esta sección de nuestro trabajo presentamos una indagación acerca de los modos en que estos estudiantes del último año utilizan estrategias de personalización o despersonalización a través del uso de las marcas de persona en sus trabajos finales. Con respecto a este punto, Muñoz y Meoniz (2018) mencionan que en la actualidad los alumnos (y también los miembros más antiguos de la academia) vacilan en la elección de mostrarse o no en los trabajos en los que comunican los resultados de sus investigaciones. Estas autoras consideran que ello puede deberse a las representaciones tradicionales dominantes sobre la escritura académica, en el sentido de caracterizarla como un discurso objetivo e impersonal sin marcas de $1^{\circ}$ persona en el que debían predominar las formas pasivas, impersonales o nominalizadas. En los últimos tiempos, esta visión ha cambiado y los estudios sobre el discurso científico y académico se inclinan por admitir que la escritura en estos ámbitos muestra marcas de subjetividad y que en sus escritos el autor dialoga con otros textos de su ámbito disciplinar, toma posición respecto a distintos puntos de vista, y a su vez, trata de convencer a sus lectores, en síntesis, asume una perspectiva retórica (Swales, 1990; Hyland, 2004; García Negroni, 2008). 
Para describir las formas que asume esta voz autoral y observar los modos en que los escritores se ponen en escena en su escritura o se 'ocultan' utilizando ciertas convenciones ya establecidas, identificamos en nuestro corpus categorías de personalización y despersonalización que son utilizadas por los estudiantes para borrar o manifestar explícitamente su presencia en el texto (Moris y Pérez, 2014). La personalización consiste básicamente en asumir la $1^{\circ}$ persona gramatical, en singular o plural, para referir al que escribe. Este uso hace evidente la presencia del productor del enunciado. La despersonalización, en cambio, que utiliza la $3^{\circ}$ persona gramatical, las oraciones impersonales u otros recursos léxicos, distancia al locutor de aquello que se dice y borra las marcas de subjetividad para presentarse como un discurso 'objetivo'.

\section{B.2. MARCAS GRAMATICALES DE PERSONA Y NO PERSONA}

En la figura 1 observamos los porcentajes de ocurrencias de las distintas estrategias de personalización y despersonalización en el corpus.

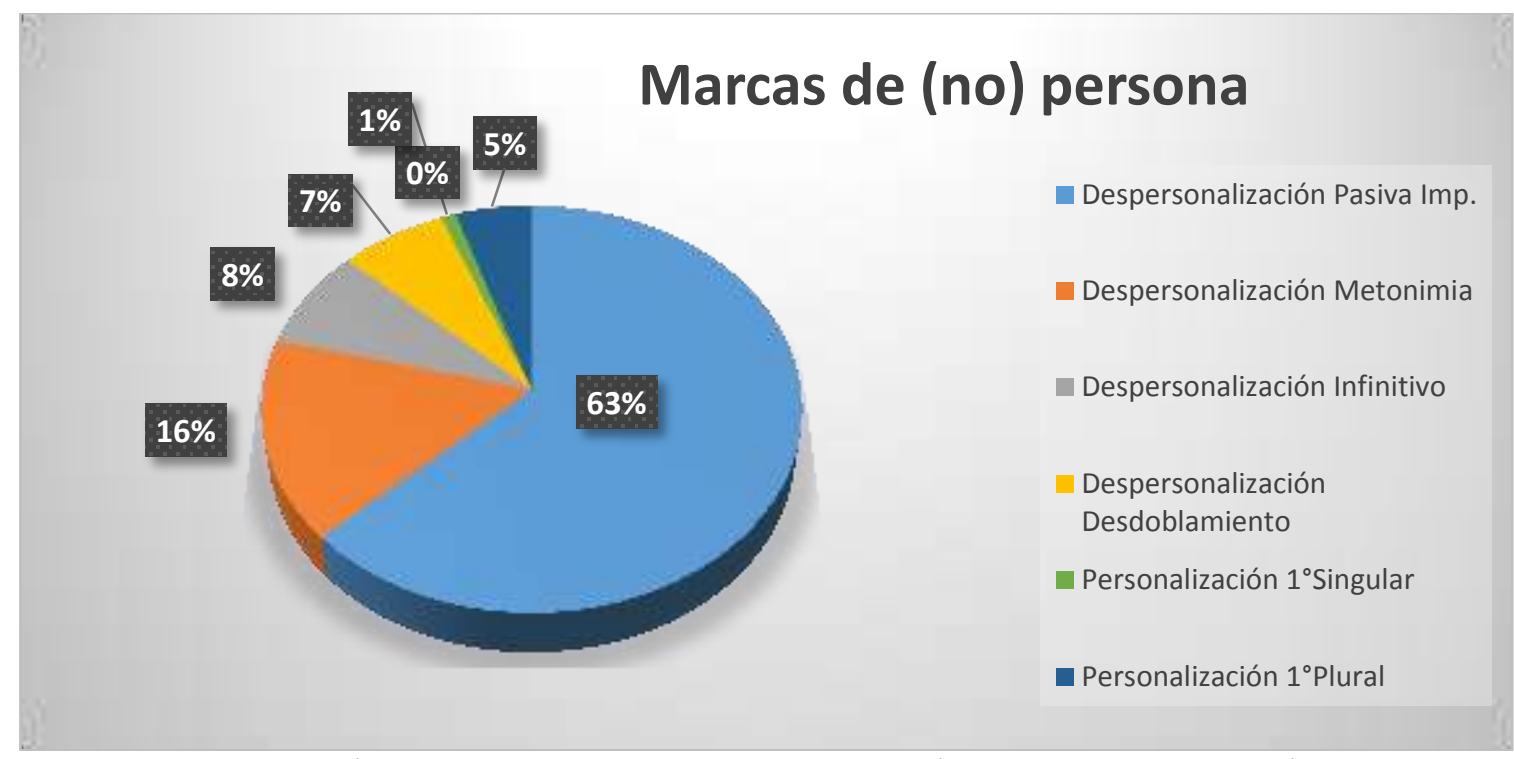

Gráfico 1. Estrategias de Personalización y Despersonalización

\section{A. DESPERSONALIZACIÓN}

En las tesinas de las cuatro carreras mencionadas, hay una tendencia a utilizar el uso de pasivas con 'se', que siguiendo a García-Negroni (2008) permiten dejar impreciso al sujeto responsable de la acción. Esta estrategia de despersonalización es la que prevalece en los trabajos académicos.

(10) El presente trabajo de investigación se enmarca en la tesina final de la Licenciatura en Trabajo Social... (TS1)

(11) Con solo indagar el aspecto gráfico de los distintos procesos electorales abordados en esta investigación se hace evidente que... (CS1)

(12) Se adoptó teórica y metodológicamente, la Geografía de la Percepción porque... (G)

(13) Se entiende el erotismo como la facultad... (L) 
También se observa en estas introducciones el uso de estructuras con infinitivo: "que neutralizan el agente del proceso evocado" (García Negroni, 2008:12). Aparece, en estos casos, siempre que se detallan los objetivos concretos del trabajo.

(14) Allí radica el interés fundamental de este trabajo: observar la campaña electoral local de 1983, poder trazar paralelismos y diferencias con el ámbito nacional y poder analizar las diferencias con los modos actuales de marketing político. (CS1)

(15) El objetivo es estudiar la percepción urbana de El Calafate para obtener el grado de legibilidad... Esto es, conocer y estudiar el grado de claridad... (G)

(16) En consecuencia, es vital profundizar y conocer nuestra realidad... (L)

Un recurso igualmente utilizado es la metonimia ${ }^{7}$ en la variante 'obra por autor'. En efecto, el enunciador no toma el rol de agente de la acción, sino que lo desplaza hacia los sustantivos que designan su producción: investigación, tesis, estudio y que funcionan como sujetos de los verbos. Es el autor quien en realidad analiza, investiga y proporciona. Sin embargo, su presencia es suprimida a través de la metonimia.

(17) Esta investigación también analizara el rol del trabajador social en su intervención... (TS2)

(18) La tesis titulada "La apropiación de las TIC entre los alumnos ingresantes a la Licenciatura en Comunicación Social de la UNPA-UARG" es un trabajo cualitativo que investiga la apropiación... (CS2)

(19) El resultado alcanzado con un estudio de esta naturaleza, proporciona una serie de herramientas muy valiosas... $(G)$

Las estrategias de impersonalización refuerzan la pretensión de neutralidad u objetividad, ocultan al sujeto de la enunciación, por lo tanto, despojan a éste de responsabilidad. En el corpus de análisis hallamos un caso de 'desdoblamiento', en el que el enunciador utiliza la tercera persona singular para referirse a sí mismo "se plantea un desdoblamiento explícito (...) Hay un uso de la $3^{\mathrm{a}}$ persona que no responde a la habitual despersonalización del autor, típica de los trabajos científicos" (Muñoz y Meoniz, 2018:7).

(20) El presente trabajo de sistematización se enfoca en el análisis de la metodología de intervención y las prácticas desarrolladas por la alumna tesista en el año 2012... (TS1)

(21) Cabe destacar que la experiencia de práctica pre-profesional a sistematizar, fue considerada una de las más importantes y significativas a lo largo del recorrido académico de la alumna tesista... (TS1)

Estos ejemplos fueron extraídos de la introducción de una tesina de Trabajo Social. Para mencionarse a sí misma, la autora se pone como complemento agente (ejemplo 20) de un participio que forma parte de un circunstancial en una construcción pasiva con 'se'. En el ejemplo 21, el enunciador "se desdibuja detrás del complemento indirecto de "recorrido académico" en vez de ser más directamente el complemento agente de "fue considerada" (Ibídem: 8).

\footnotetext{
${ }^{7}$ Para la Retórica, la metonimia es "un tropo que consiste en designar algo con el nombre de otra cosa, tomando el efecto por la causa o viceversa, el autor por sus obras, el signo por la cosa significada" (RAE).
} 


\section{B. PERSONALIZACión}

Las marcas de personalización son las marcas de persona gramatical; por lo general los alumnos escritores utilizan la primera persona singular o plural, que se presenta como una de las manifestaciones más explícitas de la subjetividad.

En las introducciones de las tesinas se nota un menor uso de la $1^{\circ}$ persona coincidente "que refiere al 'yo' cuando la producción es individual"

(22) Por lo tanto, y teniendo en cuenta la importancia de estos programas esta investigación me permitirá conocer... (TS1)

En cambio, es más frecuente el uso del llamado 'nosotros' de modestia o de autor: “(...) su referencia es precisamente el autor en el texto" (García-Negroni, 2008:14)

(23) Si bien durante la cátedra de Seminario de Tutoría de Tesina los docentes nos brindaron herramientas... (TS2)

(24) En este estudio nos remitiremos a los candidatos a Gobernador... (CS1)

(25) (...) para identificar tanto los estratos iniciales, a los que denominamos Zonas...(G)

(26) En el presente trabajo, postulamos que la poética... (L)

\section{B.3. LAS TESINAS: TENDENCIA A LAS MARCAS DE 'OBJETIVIDAD’}

En esta sección hemos indagado en el corpus de introducciones de tesinas de cuatro carreras de la UNPA, con la mirada puesta en las marcas personales. Se puede apreciar que son menos frecuentes las estrategias de personalización que las de despersonalización. Estas últimas son también más variadas en cuanto a los recursos lingüísticos que utilizan. Las estructuras más usadas son las construcciones pasivas con "se", mientras que en las primeras, se destaca el 'nosotros' de modestia. La inclinación hacia la primera de las estrategias mencionadas puede ser tomada como resultado de las exigencias de objetividad que se traducen gramaticalmente en la censura de usar la primera persona. De las tesinas estudiadas encontramos solo un caso de uso de la primera persona singular.

Habría que preguntarse si la objetividad exige necesariamente el borramiento de la primera persona, o puede descansar en otras estrategias, si el enunciador puede asumir las afirmaciones y mostrarse como fuente de lo dicho sin transgredir las normas de la comunidad académica y apoyar su objetividad en otros recursos, como pueden ser los datos obtenidos de su investigación, la solidez de sus argumentos, las referencias al saber del campo de conocimiento a través del aparato retórico de la citación bibliográfica. Por otro lado, esta exploración podría completarse con un trabajo de campo que indague acerca de las consignas e indicaciones que los docentes tutores de las tesinas realizan a sus alumnos y sobre todo acerca de las exigencias que los tribunales evaluadores imponen a estos textos. No es casual que la despersonalización sea la estrategia más usada, y que los modos en que los enunciadores aparecen en su escritura es, paradójicamente, ocultándose; esto revela que la representación dominante instaura esta restricción. Creemos que una reflexión informada en el ámbito de los talleres de tesis puede ayudar a generar una conciencia metadiscursiva de la convención que no se limite a una consigna dogmática que el escritor debe 'obedecer', sino a la consideración de todas las opciones lingüísticas disponibles para la construcción de una voz autoral. 


\section{LA INCLUSIÓN DE LAS VOCES AJENAS: GESTIONAR LAS REFERENCIAS BIBLIOGRÁFICAS}

\section{C.1. MARCO TEÓRICO}

Según Castelló “los escritores construyen 'su identidad de autor' o 'voz', mediante las conexiones dialógicas que establecen con los textos de otros autores" (2011:5) entre otras operaciones. En esta sección presentamos los resultados de la indagación en torno a los mecanismos empleados por los estudiantes para la incorporación de otras voces, es decir los modos en que manejan las citas bibliográficas.

La introducción de una tesina contiene, como decíamos anteriormente, diversas movidas retóricas (Swales, 1990; Sabaj et al, 2011): la presentación de un tema elegido por el tesista, la justificación de su importancia en el campo, el planteamiento del problema atravesado por al menos dos líneas bibliográficas en el que "se delimita el espacio de saber donde se manifiesta una carencia" (Tamola de Spiegel, 2005: 252) lo cual se investiga. Luego, los objetivos del trabajo y las características principales de la investigación. Por último, se realiza un resumen de cada parte de la tesina. Al realizar estas movidas, en especial los pasos 1.a y 1.c (ver Tabla 3) el tesista debe, casi obligatoriamente, referirse al saber ya establecido en el campo, y es aquí donde el escritor necesita apelar a la citación bibliográfica. En general, es en la escritura de esta sección donde los escritores expertos revelan su pertenencia a una comunidad disciplinar: muestran sus alianzas, sus preferencias dentro del campo, se ubican en una posición determinada e incluso negocian con otros académicos a través de la aceptación parcial de teorías, investigaciones o hipótesis. Estas habilidades de los expertos son arduamente conquistadas por los aprendices que deben apropiarse de estrategias bastante más complejas que utilizar correctamente los índices de citación.

¿Qué es una cita bibliográfica? Se trata de la reproducción de otro discurso en el propio texto (Reyes, 1993). Los modos de referirse a ellas y los tipos de referencias sirven para constatar el saber de su disciplina, por lo que, como manifiesta Correa Suárez (2016:136) "llegar a un equilibrio entre la construcción de un discurso propio y la representación de discursos ajenos sería muy importante para (...) insertarse en una comunidad disciplinar". Estos modos no solo contribuyen a la incorporación de una proposición ajena sino que también permiten expresar la posición que el autor tiene con respecto a las otras voces.

Existen diversas clasificaciones de citas bibliográficas. ${ }^{8}$ Para nuestro análisis, utilizamos las categorías textuales de citas directas (literales) e indirectas o paráfrasis (Reyes, 1993) por una parte y la distinción entre citas integradas y citas no integradas que propone Swales (1990).

La cita literal o directa repite las palabras textuales del autor y su incorporación siempre requiere marcas gráficas que establezcan el límite entre los discursos citante y citado (mediante comillas en las citas breves o un mayor sangrado para las citas más largas). Las citas indirectas o paráfrasis (también llamadas no literales) recuperan el contenido del discurso ajeno a través de reformulaciones, síntesis o comentarios, lo que no exime al enunciador de mencionar la fuente del discurso. Esta distinción coincide con las categorías de estilo directo y estilo indirecto (Massi, 2005).

Swales (1990) distingue entre citas integradas y no integradas. En las primeras se busca destacar la fuente del discurso junto con el contenido que se referencia, en ese caso el nombre

${ }^{8}$ Para una síntesis véase Sánchez Upegui (2015) 
del autor ocupa un lugar sintáctico en el texto. En las segundas el nombre del autor aparece al final de la cita, entre paréntesis o a pie de página y no forma parte del texto principal. $\mathrm{Al}$ cruzar ambas clasificaciones, obtendremos cuatro tipos de citas, que mostramos en la Tabla 6 con ejemplos extraídos del corpus

\section{Tabla 6. Tipos de citas en el corpus}

\begin{tabular}{|c|l|}
\hline $\begin{array}{c}\text { CITA DIRECTA } \\
\text { INTEGRADA }\end{array}$ & $\begin{array}{l}\text { (27) Desde la perspectiva de Héctor Schmucler (1992) "el } \\
\text { marketing político importa menos por los resultados que obtiene } \\
\text { que por los principios en que se sustenta (...) para el profesional } \\
\text { solo se trata de cumplir lo mejor posible el papel asignado al } \\
\text { servicio que presta: competir, vender" (CS) }\end{array}$ \\
CITA DIRECTA NO & $\begin{array}{l}\text { (28) La familia es concebida como "la forma de vinculación y } \\
\text { convivencia más intima en la que la mayoría de las personas } \\
\text { suelen vivir buena parte de su vida",; aunque muchos adultos } \\
\text { mayores terminen viviendo solos } \\
\text { Ander-Egg, Ezequiel; Diccionario de Trabajo Social; Editorial } \\
\text { Lumen; Buenos Aires, Argentina; pág. 127. (TS) }\end{array}$ \\
CITA INDIRECTA & $\begin{array}{l}\text { (29) García Canclini (1993) plantea el consumo como una } \\
\text { práctica cultural que abarca dimensiones económicas y también } \\
\text { aquellas no-económicas como recepción, apropiación, } \\
\text { audiencias o los usos. (CS) }\end{array}$ \\
CINTEGRADA & $\begin{array}{l}\text { (30) La noción de apropiación marca con claridad la presencia } \\
\text { de un recurso no propio pero que moviliza el desarrollo de } \\
\text { operaciones creativas para volverlo propio según reglas e } \\
\text { intereses en un escenario que es también ajeno. (Da Porta, } \\
\text { 2011). (CS2) }\end{array}$ \\
\hline INTEGRADA
\end{tabular}

Según Sánchez Upegui, las citas integradas “evidencian una mayor identificación, acuerdo y afinidad conceptual entre el escritor del texto y la fuente reportada, puesto que esta aparece en un lugar de preminencia y usualmente se le otorga una acción de reporte, que puede ser discursiva, investigativa de abstracción o cognitiva" (Sánchez Upegui, 2015: 106). Sabaj y Páez (2011) consideran que el uso de un tipo u otro configura una relación diferente entre el enunciador y el autor citado que ubican en una escala de cercanía-lejanía, en la que la directa integrada es el polo de mayor cercanía y la indirecta no integrada el de mayor lejanía entre ambos discursos.

En relación con las citas bibliográficas también observamos sus funciones textuales y retóricas. Sabaj y Páez (2011) consideran que las citas cumplen una función esencial en el entramado de la red científica y mencionan un repertorio de funciones generales: Justificar afirmaciones, Identificar metodologías, Proporcionar fundamentación, Criticar trabajos previos. Colombo y Fahler (2014) agregan algunas funciones a partir del análisis realizado en un corpus de monografías: condensadora, disparadora, epígrafe, polémica, de expansión. A partir de estas clasificaciones, rastreamos en nuestro corpus las funciones textuales y retóricas más destacadas de las citas. 


\section{C.2. TIPOS DE CITAS EN LAS INTRODUCCIONES DE TESINAS. ANÁLISIS Y DISCUSIÓN DE LOS RESULTADOS}

Como adelantamos en la metodología, realizamos una cuantificación de la cantidad de citas en el corpus de análisis, que presentamos en la tabla 7.

Tabla 7. Cantidad de citas en las Introducciones de Tesinas (por carrera)

\begin{tabular}{|l|c|c|}
\hline \multicolumn{1}{|c|}{ CARRERAS } & CANTIDAD DE TESINAS & TOTAL DE CITAS \\
\hline Lic. en Comunicación & 2 & 41 casos \\
\hline Lic. en Trabajo Social & 2 & 9 casos \\
\hline Lic. en Letras & 1 & 32 casos \\
\hline Lic. En Geografía & 1 & 26 casos \\
\hline TOTALES & 6 & 108 \\
\hline
\end{tabular}

El desequilibrio que tiene en nuestro corpus la cantidad de tesinas ${ }^{10}$ puede superarse calculando un promedio de citas por tesina; en la Tabla 8 se muestran las áreas en un orden de mayor a menor cantidad de citas (considerando el promedio). La primera observación que podemos hacer es la baja cantidad de citas en las tesinas de Trabajo Social

Tabla 8. Promedio de citas por carrera

\begin{tabular}{|l|c|}
\hline \multicolumn{1}{|c|}{ CARRERAS } & PROMEDIO DE CITAS \\
\hline Lic. en Letras & 32 \\
\hline Lic. en Geografía & 26 \\
\hline Lic. en Comunicación & 20.5 \\
\hline Lic. En Trabajo Social & 4.5 \\
\hline
\end{tabular}

A continuación, presentamos la cantidad de citas diferenciando tipos de citas directas e indirectas en el conjunto de tesinas. (Tabla 9)

Tabla 9. Citas directas e indirectas en el corpus

\begin{tabular}{|c|c|}
\hline CITAS DIRECTAS & CITAS INDIRECTAS \\
\hline 31 & 77 \\
\hline
\end{tabular}

Presentamos a continuación en la tabla 10 la cantidad de citas integradas frente a no integradas en el total del corpus. A diferencia de la clasificación anterior, puede observarse mayor paridad, no aparece una preferencia clara por un tipo de citación en relación con la integración en el cuerpo del texto.

\footnotetext{
${ }^{10}$ La diferencia en la cantidad de tesinas por carrera se debe a que las licenciaturas en Letras y Geografía son más recientes en la UNPA.
} 


\begin{tabular}{|c|c|}
\hline CITAS INTEGRADAS & CITAS NO INTEGRADAS \\
\hline 59 casos & 49 casos \\
\hline
\end{tabular}

El uso de la cita integrada tiene como función la fundamentación y justificación, en ésta, el escritor recupera los conceptos vertidos por la voz experta en el área que se investiga, por su relevancia y pertinencia (Massi, 2005), se apoya en voces autorizadas que funcionan como respaldo de una afirmación. Además da cuenta de una apropiación más acabada del discurso disciplinar por parte del enunciador. Como señalábamos en el marco teórico, el uso de citas integradas es un signo de identificación, acuerdo y afinidad conceptual entre el discurso citado y el citante (Sánchez Upegui, 2015).

En todos los casos de nuestro corpus el escritor mantiene una actitud de aceptación que se muestra a través de los verbos introductorios utilizados: plantear, decir, sostener; también a través de otras marcas de aceptación: uso de adjetivos de valoración, adverbios de modo que indican conformidad, como podemos ver en estos ejemplos (subrayado nuestro):

(31) Servato también realizó un extenso trabajo publicado en 2003, titulado "Los métodos de investigación en ciencias sociales y la producción social de conocimiento". En esta interesante publicación la autora realiza un análisis exhaustivo desde distintos enfoques metodológicos... (TS2)

(32) En este sentido la poesía puede verse como sostiene Blas Matamoro "un simulacro bélico, como una batalla anacrónica (2002:197)” (L)

La cita no integrada puede funcionar como definición a la que después el escritor se opone y ofrece su punto de vista (33), puede ser metodológica, es decir que explicite metodologías o procedimientos que el tesista utilizará en su análisis (34), puede servir de apoyo, como en el caso de (35), de fundamentación (36) y de justificación de una afirmación como en el caso (37).

(33) La familia es concebida como "la forma de vinculación y convivencia más íntima en la que la mayoría de las personas suelen vivir buena parte de su vida"11; aunque muchos adultos mayores terminen viviendo solos. ${ }^{l}$

Ander-Egg, Ezequiel; Diccionario de Trabajo Social; Editorial Lumen; Buenos Aires, Argentina; pág. 127. (TS)

(34) Las entrevistas abiertas, se fundamentan en una guía general de contenido y el entrevistador posee toda la flexibilidad para manejarla (él maneja el ritmo, la estructura y el contenido de los ítems). ${ }^{l}$

1 Hernández Sampieri, R; C.F Collado y P.B Lucio (2006); Metodología de la Investigación Social; Cuarta Edición; Editorial McGraw-Hill; D.F, México; pág.597. (TS)

(35) Sobre lo que la gente dice o hace, es producto del modo en el que se defina su mundo (Taylor y Bogdam: 1992,23). (TS2)

(36) Esas desigualdades están dadas por, como se dijo anteriormente, la posibilidad de acceso a la tecnología, pero también por las posibilidades de desarrollar habilidades y competencia instrumental es decir, esa capacidad para aprovechar adecuadamente las posibilidades que ofrecen las nuevas tecnologías (Bañón, 2010). (CS2)

(37) En consecuencia, es vital profundizar y conocer nuestra realidad contada por sus propios actores de la cual nos sentimos identificados al compartir una misma historia porque "una 
crítica latinoamericana sólo podrá ser tal en la medida en que se encuentre articulada a la historia y proyecto cultural latinoamericano (Osorio Tejada 2006:6) (L)

En el entrecruzamiento de los conceptos de citas integradas/ no integradas con citas de estilo directo e indirecto obtuvimos el siguiente cuadro (Tabla 11).

Tabla 11. Citas directas, indirectas/integradas, no integradas en el corpus

\begin{tabular}{|c|c|c|c|c|}
\hline TESINAS & $\begin{array}{c}\text { DIRECTAS } \\
\text { INTEGRADAS }\end{array}$ & $\begin{array}{c}\text { DIRECTAS } \\
\text { NO } \\
\text { (+ cercanía) }\end{array}$ & $\begin{array}{c}\text { INDIRECTAS } \\
\text { INTEGRADAS }\end{array}$ & $\begin{array}{c}\text { INDIRECTAS } \\
\text { NO } \\
\text { INTEGRADAS } \\
\text { (+lejanía) }\end{array}$ \\
\hline TOTALES & 21 & 11 & 37 & 39 \\
\hline
\end{tabular}

Parece haber una tendencia mayoritaria al uso de citas indirectas, tanto integradas como no integradas. Un aspecto interesante es la relación de cercanía y lejanía que se establece entre el autor de la cita y el enunciador de la investigación (Sabaj \& Páez, 2011). Una forma de esquematizarlo, fue señalar en la tabla 11 mayor cercanía del lado izquierdo del margen, mientras que en el lado derecho se observa mayor lejanía entre autor de la cita y escritor. Castelló et al (2012) sostienen que tanto entre estudiantes de nivel secundario como entre universitarios, priman las citas directas y destacan que la incorporación de citas indirectas, sobre todo no integradas, constituye un gran desafío incluso para estudiantes de doctorado. Es de destacar que en nuestro corpus los estudiantes escritores parecen dominar el uso de citas indirectas, tanto integradas como no integradas.

Nos preguntamos a continuación si las diferentes áreas disciplinares muestran alguna característica particular al respecto, por lo que observamos el uso que cada disciplina hace de los cuatro tipos de citas. (Tabla 12 y Gráfico 2)

Tabla 12. Tipos de citas por carreras expresadas en porcentajes

\begin{tabular}{|c|c|c|c|c|}
\hline Carreras & $\begin{array}{c}\text { Directas } \\
\text { integradas }\end{array}$ & $\begin{array}{c}\text { Directas no } \\
\text { integradas }\end{array}$ & $\begin{array}{c}\text { Indirectas } \\
\text { integradas }\end{array}$ & $\begin{array}{c}\text { Indirectas no } \\
\text { integradas }\end{array}$ \\
\hline $\begin{array}{c}\text { Comunicación } \\
\text { Social }\end{array}$ & 27 & 12 & 34 & 26.8 \\
\hline Trabajo Social & 0 & 33 & 0 & 66 \\
\hline Letras & 21 & 6.25 & 68.75 & 3.12 \\
\hline Geografía & 11.5 & 3.84 & 3.84 & 80.76 \\
\hline
\end{tabular}




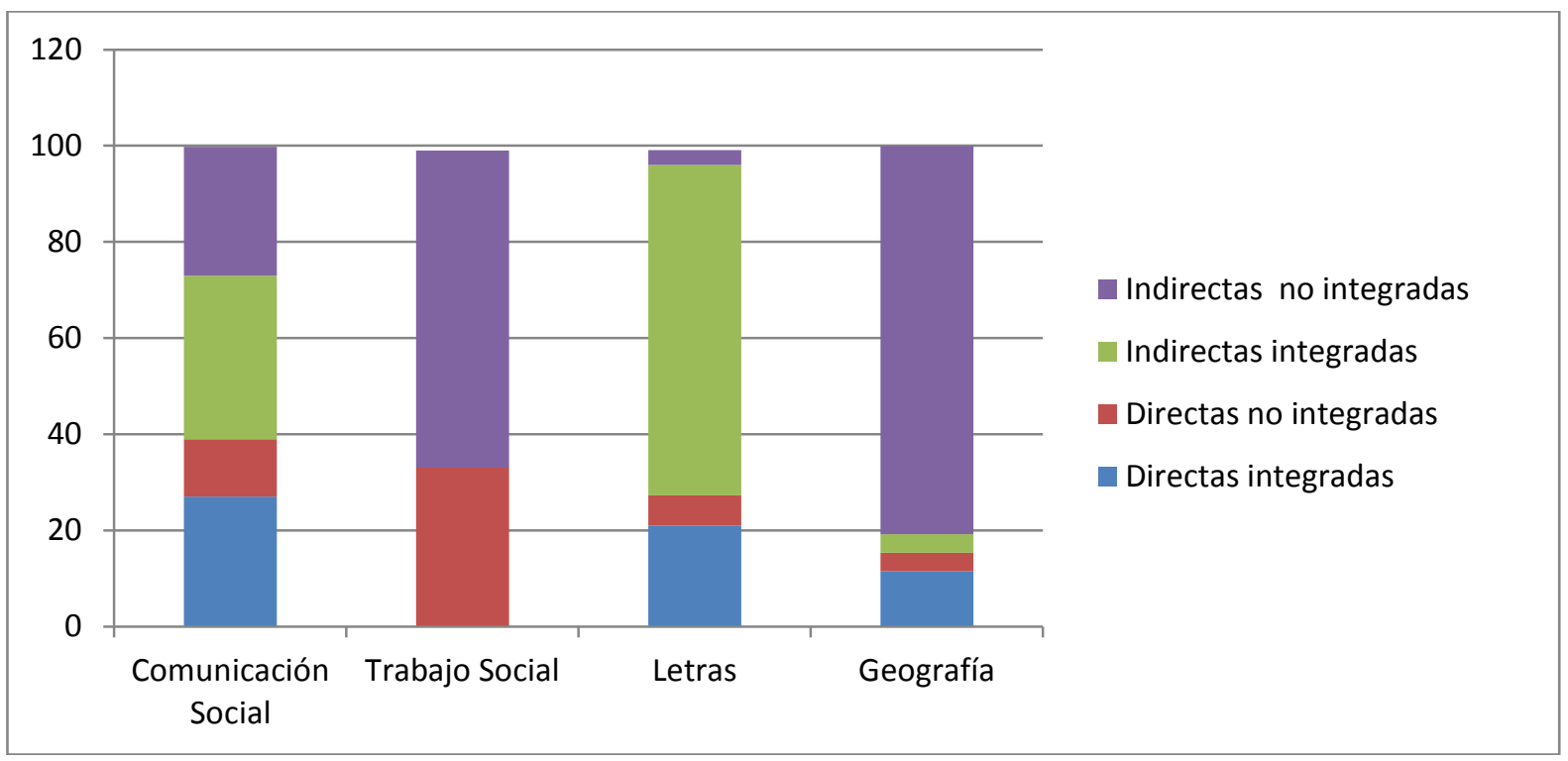

Gráfico 2. Tipos de citas por carreras (expresadas en porcentaje)

Podemos observar que tanto Letras como Comunicación Social se inclinan por la cita indirecta integrada, mientras que en Trabajo Social y Geografía prevalece la cita indirecta no integrada. De todos modos, se destaca en las cuatro áreas el estilo indirecto (aunque recordemos la baja frecuencia de citación del área de TS), lo que puede revelar una apropiación mayor del discurso disciplinar, ya que el enunciador hace suyos los conceptos, las ideas del discurso citado reformulándolas por medio de paráfrasis, mientras que las citas directas muestran claramente la frontera entre el texto citado y el citante. Este último tipo de citación señala un movimiento de apelación al saber ya construido aunque evidencia ese límite entre un discurso ya producido en otra situación y el propio discurso.

Distinto es el caso de las citas no integradas, que funciona más como estrategia de distanciamiento, en la que se nota una ausencia de mención directa de las fuentes bibliográficas. Esto pudo observarse sobre todo en el área de Trabajo Social, en la que el autor de la cita queda relegado, en la mayoría de los casos, a la nota al pie. El uso frecuente de la cita no integrada en esta carrera, puede deberse a la búsqueda de un efecto de neutralidad u objetividad, donde el foco está más en las ideas que en las personas puntuales que las produjeron. Además, observamos que Trabajo Social es la carrera que menos cita, lo que también puede estar ligado a lo dicho anteriormente. Lo mismo pudo observarse en Geografía, donde de 26 casos, 21 son de este tipo.

\section{CONCLUSIONES GENERALES}

Este trabajo de investigación nos permitió identificar algunas de las estrategias discursivas con que los escritores noveles intentan dar forma a una voz propia para dar a conocer sus trabajos en cuatro áreas específicas de conocimiento. Entre los recursos lingüísticos que los escritores noveles utilizan para construir una voz propia que Castelló (2011) identifica, elegimos tres para indagar en las Introducciones de tesinas de cuatro carreras de la UNPA: en relación con los aspectos estructurales, que conciernen a la organización de las Introducciones, analizamos los Movimientos retóricos a través de los cuales los autores muestran la conexión entre sus propias ideas y hallazgos con los objetivos generales del campo científico al que pertenecen, señalan la importancia de su estudio particular y justifican sus puntos de vista y opciones de investigación. En relación con el posicionamiento, 
seleccionamos las marcas de autorreferencia, a través de las cuales los escritores regulan su visibilidad en el texto. Estos rasgos señalan el grado de presencia explícita en la escritura fundamentalmente a través de la frecuencia de uso (o de la ausencia) de la primera persona. Según Hyland (2005) el uso de la primera persona es una elección consciente de los expertos para adoptar una identidad situada disciplinarmente (2005: 181). En relación con el diálogo intertextual analizamos los modos en que el escritor vincula su propio enunciado con las voces del campo al que pertenece: si lo hace de manera literal o reformulando sus aportes, si lo hace integrando la fuente a su propio discurso o manteniendo cierta distancia con él.

Nuestros resultados muestran que, en relación con la organización retórica aparecen en las introducciones los pasos correspondientes al movimiento Ocupar el nicho de investigación fundamentalmente a través de la formulación de objetivos y de preguntas y son escasas las textualizaciones que apuntan a Presentar o Establecer el campo de investigación y Establecer el espacio vacante o Identificar un nicho de investigación. En el caso de las tesinas de TS estos dos movimientos directamente no aparecen. Como lo señalábamos en la sección 1, esta estructura retórica incompleta tiene el efecto de que el trabajo de tesis aparece vinculado débilmente con la corriente general del campo de conocimiento. Este rasgo además puede señalarse como característico de cierta 'ignorancia' del escritor acerca de su responsabilidad para indicar la importancia de su propia investigación (como un aporte a la disciplina) y para conducir al lector de lo general a lo particular.

En relación con las estrategias de personalización, el estilo de las introducciones tiende a ser impersonal, a través del uso de una variedad de recursos: estructuras pasivas con 'se', infinitivos, recurso a la metonimia. Cuando el tesista usa la primera persona lo hace en plural, con el 'nosotros' de modestia. Hay sólo una ocurrencia de uso de $1^{\circ}$ persona singular o persona coincidente (ya que todas las tesinas son de un único autor). Creemos que existe una relación entre la exigencia de objetividad que pesa sobre el discurso académico y esta tendencia a la despersonalización. En ocasiones la exigencia de borramiento tensiona sobre el escritor de manera tal que cuando no puede evitar la referencia a sí mismo, usa la $3^{\circ}$ persona. Estos resultados podrían completarse con un análisis completo de las tesinas y el estudio de las regulaciones que los instructivos y las consignas con que los equipos de cátedra orientan la escritura. A la vez, sería interesante añadir información que recupere parte de las experiencias de los alumnos tesistas en relación con las dificultades (o facilidades) que enfrentaron al redactar estos trabajos finales.

Por último, la indagación acerca de los modos en que el escritor dialoga con las voces del campo mostró que estos escritores noveles utilizan una gran variedad de recursos de citación, aunque con diferencias según las disciplinas, ya que las tesinas de TS muestran muy pocas citas en comparación con las otras tres carreras. A este dato hay que agregar que en esta disciplina el autor de la cita queda relegado, en la mayoría de los casos, a la nota al pie. Este uso puede ponerse en relación con la despersonalización señalada más arriba ya que podría obedecer a la búsqueda de un efecto de neutralidad u objetividad, donde el foco está más en las ideas que en las personas puntuales que las produjeron. En las otras tres áreas se destaca el uso del estilo indirecto lo que puede revelar una apropiación mayor del discurso disciplinar, ya que el enunciador hace suyos los conceptos, las ideas del discurso citado reformulándolas por medio de paráfrasis. Si relacionamos estos resultados con las observaciones realizadas más arriba acerca de los movimientos retóricos, creemos que una orientación didáctica debería mostrar la vinculación que existe entre los dos primeros movimientos retóricos y la citación bibliográfica. De esta manera, las citas podrían contribuir a delinear los contornos de una zona del campo de investigación y revelar los lazos con el trabajo particular que realizó el 
tesista. Es posible que los escritores no sean conscientes de la diferencia retórica entre una cita integrada al propio discurso y una cita cuyo autor queda relegado en una nota al pie, estamos convencidas de que la explicitación de estos matices puede contribuir a crear mayor conciencia metadiscursiva tanto entre los alumnos como entre los profesores.

Sería necesario completar estas indagaciones extendiendo la mirada hacia el resto de las secciones de las tesinas como también ampliar el número de tesinas del corpus para corroborar o no estas primeras observaciones. Si contáramos además con un corpus digital ${ }^{12}$ sería posible por un lado, integrar mayor cantidad de textos (y más carreras) y por otro, aplicar herramientas de análisis digitales para análisis del léxico y las formulaciones sintácticas.

Por último, queremos enfatizar nuestra perspectiva acerca de que la escritura académica de los estudiantes es una práctica social en el sentido de que quienes escriben están aprendiendo no solo a comunicarse de modos particulares sino que están aprendiendo cómo ser tipos particulares de personas, es decir a escribir como académicos, como geógrafos, como científicos sociales. Por lo tanto, la escritura académica concierne también a la identidad personal y social (Curry y Lillis, 2003).

\section{BIBLIOGRAFÍA}

AMOSSY, R. (ed.) (1999). Images de soi dans le discours - La construction de l'ethos, Lausanne: Delachaux et Niestlé.

BUNTON, David (2002). Generic moves in Ph.D. thesis introductions. En: John Flowerdew. Academic discourse (pp. 57-75). London: Longman.

CASTELLÓ, M. (2009). Aprender a escribir textos académicos: ¿copistas, escribas, compiladores o escritores? En Pozo et al. Psicología del aprendizaje universitario. La formación en competencias. Madrid: Morata (p.120 a 133)

CASTELLÓ, M. (2011). La voz del autor en la escritura académica: Una propuesta para su análisis. Revista Signos, 44(76) 105-117. https://doi.org/10.4067/S0718-09342011000200001

CASTELLÓ, M (2013). La escritura académica en la universidad. En Revista de Docencia Universitaria Vol.11(1) p.17-36. https://doi.org/10.4995/redu.2013.5590

CASTElló, M. BAÑAleS, G., CORCELlES, M., IÑNESTA, A. y VEGA, N. (2012). La voz del autor en los textos académicos: construyendo la identidad como escritor. C. Monereo y J. I. Pozo (Eds.), La identidad en Psicología de la Educación: enfoques actuales, utilidad y límites. Barcelona: Edebé, 2012.

COLOMBO, M.V. y FAHLER, M.V. (2014). Estrategias de inclusión de voces en monografías de estudiantes de Letras e Historia (UBA). Actas del Congreso Nacional Cátedra UNESCO. UNRosario.

CORREA SUÁREZ, H. (2016). La voz de los estudiantes de grado en trabajos monográficos de tres carreras de humanidades: las representaciones del discurso ajeno y el discurso propio en Gabbiani, B, y Orlando, V. (2016). Escritura, lectura y argumentación en las monografías de Humanidades. Montevideo: U de la R.

\footnotetext{
${ }^{12}$ Confiamos en que pronto sea una realidad el Repositorio UNPA donde podremos consultar online las principales producciones que se producen en la Universidad.
} 
CURRY, M. y LILLIS, T. (2003). Issues in academic writing in higher education. En Coffin, C. et al.Teaching academic writing: A toolkit for higher education. London: Routledge, 2005.

DIFABIO DE ANGLAT, Hilda; ÁLVAREZ, Guadalupe (2015). Apuntes de cátedra: Estrategias para la producción conceptual y escrita en el posgrado en Ciencias sociales y humanas. Universidad Nacional de Cuyo.

DI STÉFANO, M. (2017). La enseñanza de la escritura como crítica: un abordaje glotopolítico. Ponencia presentada en el III Congreso Latinoamericano de Glotopolítica Reflexiones desde y hacia América y Europa. Hannover, (27-30/9).

GABBIANI, B, y ORLANDO, V. (2016). Escritura, lectura y argumentación en las monografías de Humanidades. Montevideo: U de la R.

GARCÍA NEGRONI, M. (2008). Subjetividad y discurso científico-académico. Acerca de algunas manifestaciones de la subjetividad en el artículo de investigación en español. Revista Signos 41(66).

HYLAND, K. (2004). Disciplinary Discourses. Social interactions in Academic Writing. Ann Arbor: Michigan Classics Edition.

HYLAND, K (2002) Authority and invisibility: Authorial identity in academic writing. Journal of Pragmatics. 34(8), 1091-1112. https://doi.org/10.1016/S0378-2166(02)00035-8

JARA SOLAR, Iván (2013). Descripción funcional de introducciones de tesis doctorales en las disciplinas de química y lingüística. Onomázein, 28, pp. 72-87. https://doi.org/10.7764/onomazein.28.7

MAINGUENEAU, D. (2002). Problèmes d'ethos, en Pratiques $N^{\circ} 113 / 114$, junio de 2002, pp. 55-67. (Traducido y seleccionado por M. Eugenia Contursi). https://doi.org/10.3406/prati.2002.1945

MASSI, M. (2005). Las citas en la comunicación académica escrita en Revista iberoamericana de Educación 36(5).

MORIS, J.P. y PÉREZ, G. (2014) La Monografía, en Navarro, F. (2014) Manual de escritura para carreras de Humanidades. Bs. As., FiloUBA. Cap. 4

MUÑOZ, N. y MEONIZ, S. (2018). Alternancia de posiciones enunciativas en la escritura académica de estudiantes de Licenciatura en Trabajo Social. Ponencia presentada en las X Jornadas de Letras. UNPA-UARG.

NAVARRO, F. y MORIS, J.P. (2012) Estudio contrastivo de monografías escritas en las carreras de Educación, Filosofía, Historia y Letras en Bosio, I.; Castel, V.; Ciapuscio, G.; Cubo, L. y Müller, G. Discurso especializado: estudios teóricos y aplicados. Volúmenes temáticos de la SAL. Mendoza, FyL UNCuyo.

PUIATTI DE GOMEZ, H. y CASTRO DE CASTILLO, E. (2011) Escribir la Introducción de la tesis, en Cubo-Lacon-Puiatti, Escribir una tesis. Córdoba: Comunicarte. Cap. 2.

REYES, G. (1995). Los procedimientos de cita: estilo directo y estilo indirecto. España, Madrid, Arco Libros.

RIERA, G. (2015) Tensiones y conflictos en las concepciones y programas de escritura académica. En Traslaciones. Revista Latinoamericana de Lectura y Escritura. Vol. 2 (3) Julio 2015, pp. 127-152.

SABAJ MERUANE, O., TORO TENGROVE, P. y FUENTES CORTÉS, M. (2011). Construcción de un modelo de movidas retóricas para el análisis de artículos de investigación en español. Onomazein 24 (2011/2), pp. 245-271.

SABAJ \& PÁEZ (2010) Tipos y funciones de las citas en artículos de investigación de tres disciplinas en Literatura y Lingüística $N^{\circ} 22$, pp. 117-134.

https://doi.org/10.4067/S0716-58112010000200009 
SANCHEZ Upegui (2015) Análisis lingüístico de la citación en artículos de ciencias sociales y humanas en Revista Lasallista de investigación Vol-12 $N^{\circ} 1$, pp. 99-124. https://doi.org/10.22507/rli.v12n1a10

SWALES, J. (1990). Genre Analysis. English in academic and research settings. Cambridge: Cambridge University Press.

TAMOLA DE SPIEGEL, Diana (2005) en Cubo de Severino, L. La tesina de licenciatura en Los textos de la Ciencia, principales clases del discurso académico-científico. Cap. 2 pp. 235-259. Córdoba: Comunicarte. 